\title{
Editorial
}

\section{Innovative Micro- and Nanostructured Materials and Devices for Energy Applications}

\author{
Luciano Mescia, ${ }^{1}$ Andrea Chiappini, ${ }^{2}$ Alessandro Massaro, ${ }^{3}$ and Shivakiran N. B. Bhaktha ${ }^{4}$ \\ ${ }^{1}$ Dipartimento di Ingegneria Elettrica e dell'Informazione (DEI), Politecnico di Bari, Via E. Orabona 4, 70125 Bari, Italy \\ ${ }^{2}$ Consiglio Nazionale delle Ricerche-Istituto di Fotonica e Nanotecnologie (CNRIFN), Via alla Cascata 56/C, 38123 Trento, Italy \\ ${ }^{3}$ Istituto Italiano di Tecnologia (IIT), Center for Biomolecular Nanotechnologies (CBN), Via Barsanti 1, 73010 Arnesano, Italy \\ ${ }^{4}$ Department of Physics and Meteorology, Indian Institute of Technology Kharagpur, Kharagpur 721302, India
}

Correspondence should be addressed to Luciano Mescia; mescia@deemail.poliba.it

Received 5 December 2013; Accepted 5 December 2013; Published 13 August 2014

Copyright (C) 2014 Luciano Mescia et al. This is an open access article distributed under the Creative Commons Attribution License, which permits unrestricted use, distribution, and reproduction in any medium, provided the original work is properly cited.

During the last decades a lot of research activities in the field of complex materials have been accomplished and today there are a wide range of applications where these materials are used. In particular, micro- and nano-structured materials are essential to develop (i) high performance sensors for environmental monitoring and industrial applications, (ii) novel energy harvesting materials and systems to be used for converting solar, thermal and mechanical energy into electrical energy, and (iii) novel electrical insulator for high voltage applications, (iv) as well as to improve the efficiencies and market penetration of inorganic photovoltaics. Moreover, the advances in micro- and nano-structured materials, enabling novel, powerful, and exciting applications, are based on research results arising from different investigation fields. This special issue is an example of these interdisciplinary contributions in using synthesis, modeling, and characterization techniques of materials as well as the related design and optimization of innovative components and functional devices for enabling the future energy applications.

In the paper entitled "Hydrothermal synthesis of Pt-, Feand $\mathrm{Zn}$-doped $\mathrm{SnO}_{2}$ nanospheres and carbon monoxide sensing properties" the authors illustrate a simple hydrothermal method to synthetize $\mathrm{M}$-doped $(\mathrm{M}=\mathrm{Pt}$, Fe, and $\mathrm{Zn}) \mathrm{SnO}_{2}$ nanospheres for high-performance $\mathrm{CO}$ gas sensors. In particular, they demonstrated that the Pt-doped $\mathrm{SnO}_{2}$ nanospheres sensor exhibit significantly enhanced CO sensing performance in terms of sensitivity, operating temperature, selectivity, response and recovery time, and reproducibility.

The paper "Analytical optimization of piezoelectric circular diaphragm generator" describes analytical study of the piezoelectric circular diaphragm microgenerator. The authors illustrate results for PZT and PMN-PT piezoelectric materials with steel and aluminum substrates and demonstrate that optimal value for the radius and thickness of the piezoelectric layer can be used to optimize the produced electrical energy from mechanical pressure.

The paper "CFD study on wall/nanoparticle interaction in nanofluids convective heat transfer" illustrates that Brownian motion can be considered as one of the main factor for enhancing forced convective heat transfer coefficient of nanofluids. The simulation results clearly indicate that microconvection/mixing induced by the Brownian motion of nanoparticles could significantly affect the macroconvective heat transfer capability of the nanofluids.

In the paper "Electrospun polymer-fiber solar cell" the authors show a novel electrospun polymer-fiber solar cell synthesized by electrospinning. In particular, the measured red shift in the absorption and photoluminescence for the MEH-PPV polymer fibers may offer promising prompts for increasing the device efficiency compared to thin films. Moreover, the authors highlight that the device structure may 
bring significant impact in the field of organic photovoltaic if proper adjustments for electrospun polymer-fiber solar cells are made.

\section{Acknowledgment}

The guest editors thank all the authors and reviewers for their valuable contribution.

Luciano Mescia Andrea Chiappini Alessandro Massaro Shivakiran N. B. Bhaktha 

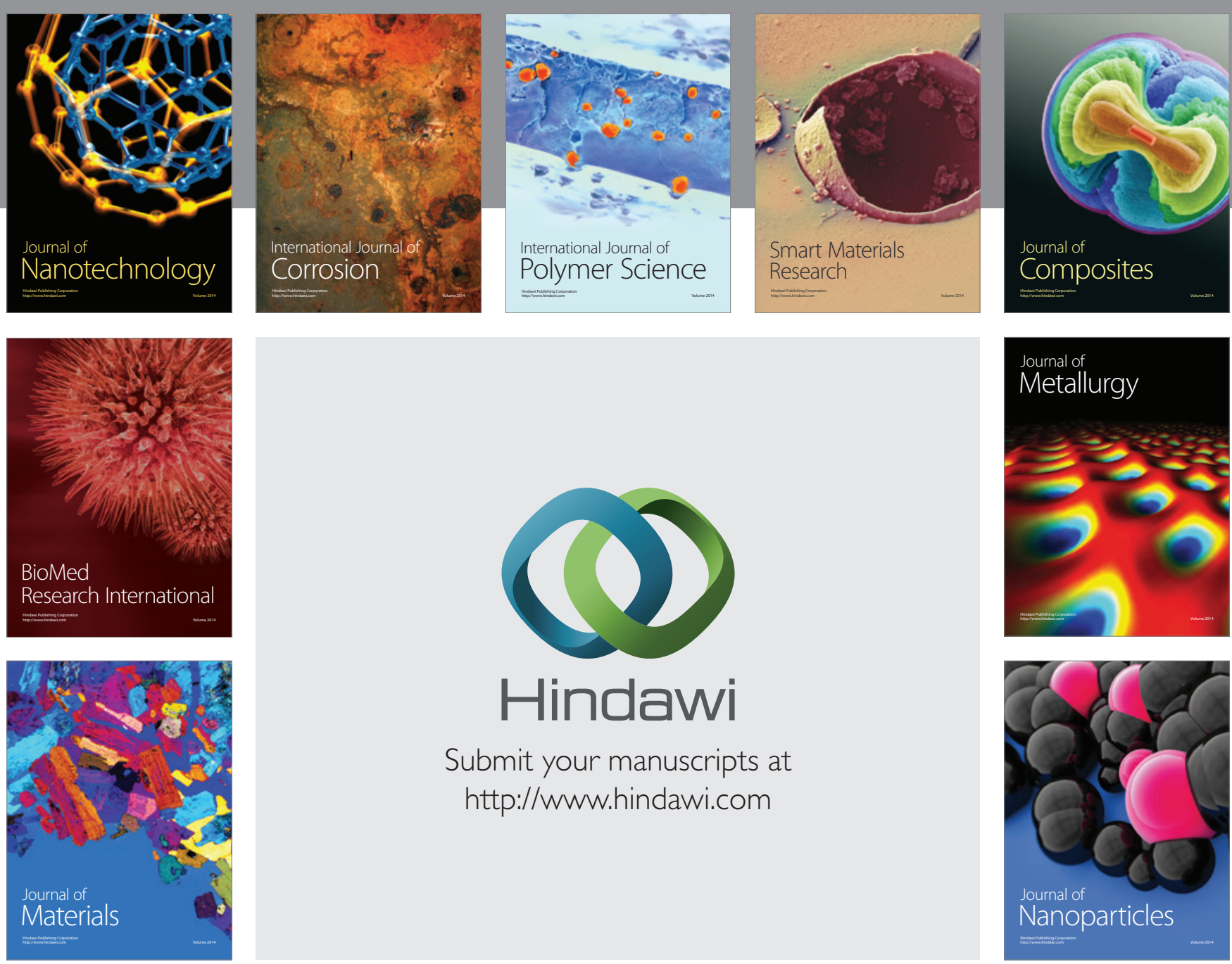

Submit your manuscripts at http://www.hindawi.com
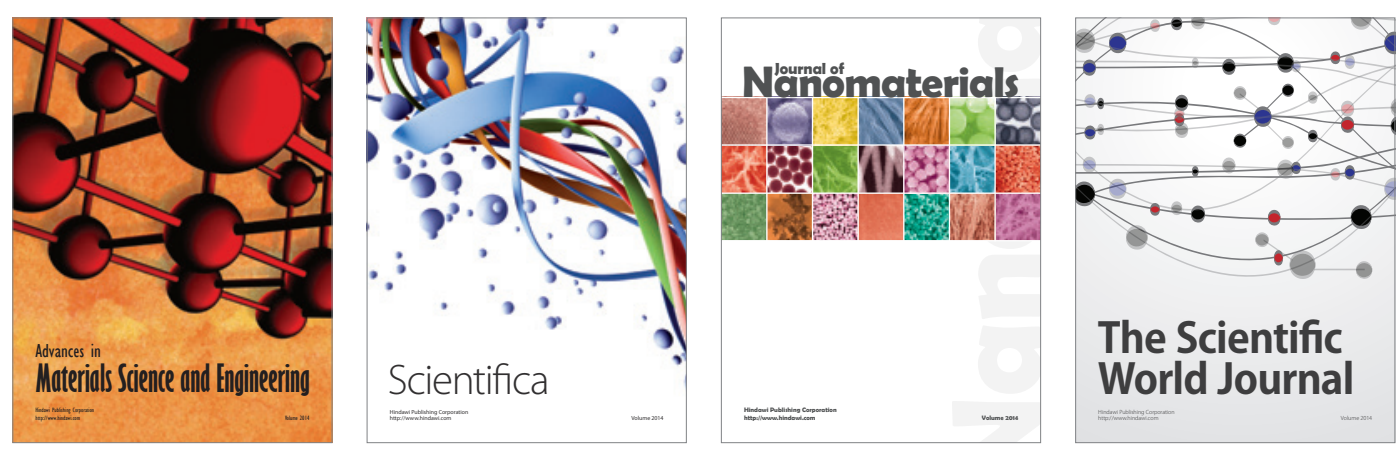

\section{The Scientific World Journal}
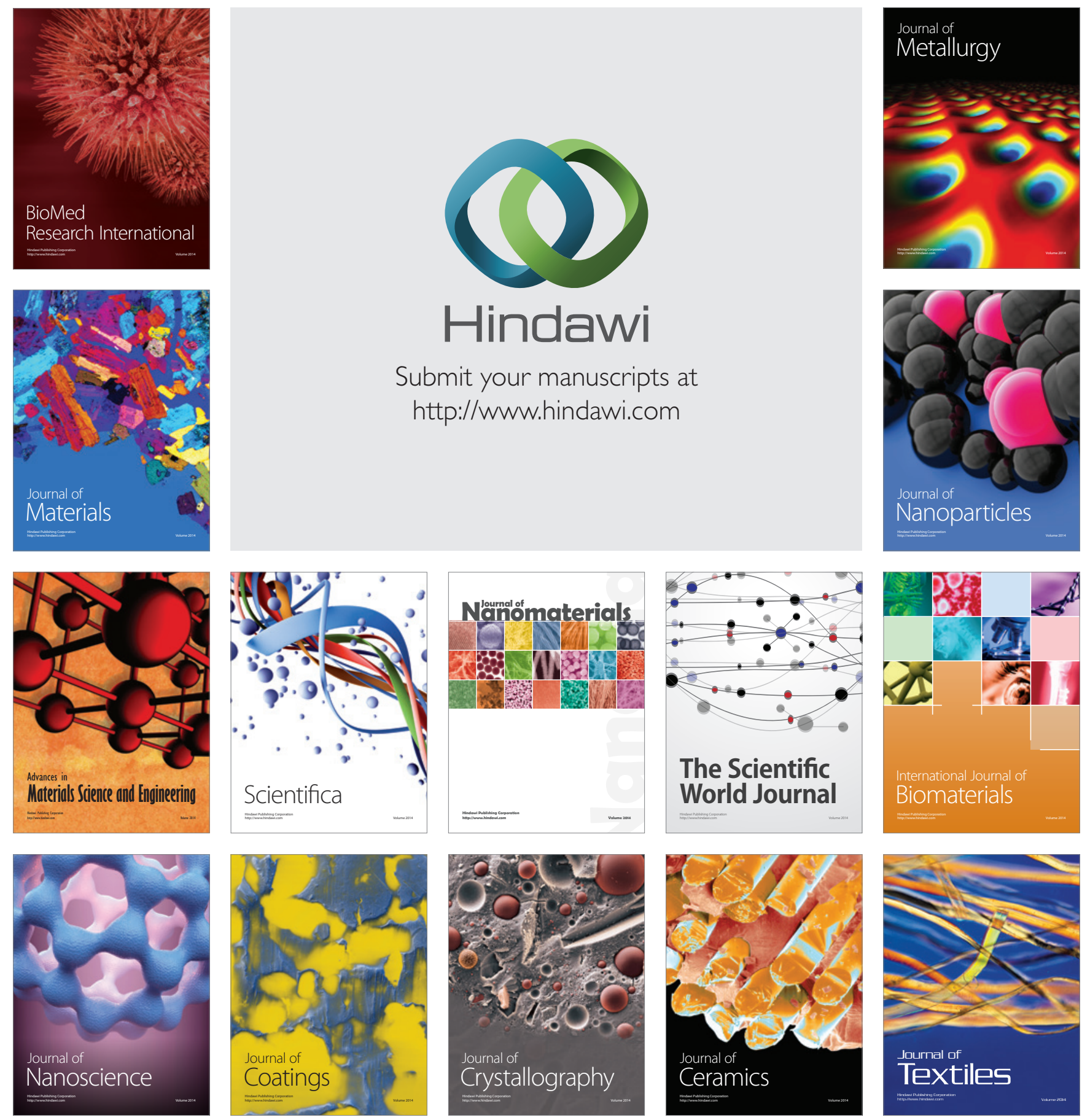